\title{
Electric Field Control of Spins in Molecular Magnets
}

\author{
Junjie Liu, ${ }^{1,}{ }^{*}$ Jakub Mrozek, ${ }^{1}$ William K. Myers, ${ }^{2}$ Grigore A. Timco, ${ }^{3}$ Richard E. P. Winpenny, ${ }^{3}$ \\ Benjamin Kintzel, ${ }^{4}$ Winfried Plass, ${ }^{4}$ and Arzhang Ardavan ${ }^{1, \dagger}$ \\ ${ }^{1}$ CAESR, Department of Physics, University of Oxford, The Clarendon Laboratory, Parks Road, Oxford OXI 3PU, United Kingdom \\ ${ }^{2}$ CAESR, Inorganic Chemistry Laboratory, University of Oxford, South Parks Road, Oxford OX1 3QR, United Kingdom \\ ${ }^{3}$ School of Chemistry and Photon Science Institute, The University of Manchester, Manchester, M13 9PL, United Kingdom \\ ${ }^{4}$ Institut für Anorganische und Analytische Chemie, Friedrich-Schiller-Universität Jena, Humboldtstraße 8, 07743 Jena, Germany
}

(Received 21 August 2018; published 25 January 2019)

\begin{abstract}
Coherent control of individual molecular spins in nanodevices is a pivotal prerequisite for fulfilling the potential promised by molecular spintronics. By applying electric field pulses during time-resolved electron spin resonance measurements, we measure the sensitivity of the spin in several antiferromagnetic molecular nanomagnets to external electric fields. We find a linear electric field dependence of the spin states in $\mathrm{Cr}_{7} \mathrm{Mn}$, an antiferromagnetic ring with a ground-state spin of $S=1$, and in a frustrated $\mathrm{Cu}_{3}$ triangle, both with coefficients of about $2 \mathrm{rad} \mathrm{s}^{-1} / \mathrm{V} \mathrm{m}^{-1}$. Conversely, the antiferromagnetic ring $\mathrm{Cr}_{7} \mathrm{Ni}$, isomorphic with $\mathrm{Cr}_{7} \mathrm{Mn}$ but with $S=1 / 2$, does not exhibit a detectable effect. We propose that the spinelectric field coupling may be used for selectively controlling individual molecules embedded in nanodevices.
\end{abstract}

DOI: 10.1103/PhysRevLett.122.037202

Among the physical manifestations of electronic quantum spins, molecular systems exhibit a range of advantages for technological applications: molecular spin properties may be tailored chemically for particular purposes while retaining substantial quantum coherence times [1-5]; supramolecular chemical synthesis offers routes to complex multispin structures [6,7]; and the principles by which individual molecular spin states may be interrogated have been demonstrated [8-11]. These achievements represent significant progress towards realizing the promise of molecular quantum spintronics [12-15].

The canonical approach to manipulating molecular spins exploits the Zeeman interaction between an oscillatory magnetic field and electron spins [16]. However, this approach to controlling individual molecular components in an integrated device is challenging because it is difficult to localize magnetic fields with the spatial resolution required (on the scale of $1 \mathrm{~nm}$ ), and undesirable cross talk is inevitable. On the other hand, electrically controllable molecular spins [17-20] would offer significant architectural advantages: strong electric fields ( $E$ fields) can be generated and localized over small length scales, and it has been shown that projective spin measurements may be achieved using the same electrodes as for coherent spin manipulation $[9,21,22]$. However, the challenge of quantifying $E$-field coupling to spins in molecular nanomagnets persists. Unlike charges, localized electron spins couple only weakly to $E$ fields because spin-orbital interactions scale with the size of orbitals $L$ as $L^{3}$ [23]. This motivates proposals to exploit $E$-field effects in antiferromagnetically coupled molecular nanomagnets, in which the
$E$-field-induced modifications to exchange interactions can lead to enhanced spin-electric couplings $[18,19]$.

Study of the effect of $E$ fields applied to spins in ESR experiments has, in the past, been used as a means of investigating the symmetry of transition metal centers in insulators or organic materials (e.g., proteins and enzymes) [24]; a first-order dependence of the spin energy levels on the applied $E$ field is indicative of an inversion-symmetrybroken environment. These techniques were later applied to manipulate coherently potential spin qubits in semiconductors [25] and to study $E$-field-induced decoherence $[26,27]$. More recently, progress has been made in directly applying high-frequency oscillatory $E$ fields to drive the spins of individual atoms on surfaces [28], and molecular spins in single-molecule transistors $[9,22]$.

In this Letter we investigate the spin-electric coupling in a selection of antiferromagnetic molecular nanomagnets, by introducing $E$-field pulses to frozen solutions of the molecules during ESR Hahn-echo sequences. Our experiment provides a general method for screening for spinelectric couplings in molecular magnets, thus paving the way for implementation of $E$-field control in molecular spintronics.

We studied three antiferromagnetic molecules. $\mathrm{Cr}_{7} \mathrm{Ni}$ and $\mathrm{Cr}_{7} \mathrm{Mn}[1,2,29,30]$, shown in Fig. 1(a), share the same molecular structure of a ring formed of seven $\mathrm{Cr}$ atoms and a heteroatom (either $\mathrm{Ni}$ or $\mathrm{Mn}$ ), bridged by carboxylate ligands [31]. The antiferromagnetic coupling leads to welldefined magnetic ground states below about $10 \mathrm{~K}$, with a total spin of $S=1$ for $\mathrm{Cr}_{7} \mathrm{Mn}$ and $S=1 / 2$ for $\mathrm{Cr}_{7} \mathrm{Ni}$. The peripheral pivalate groups are deuterated and the molecules 
(a)

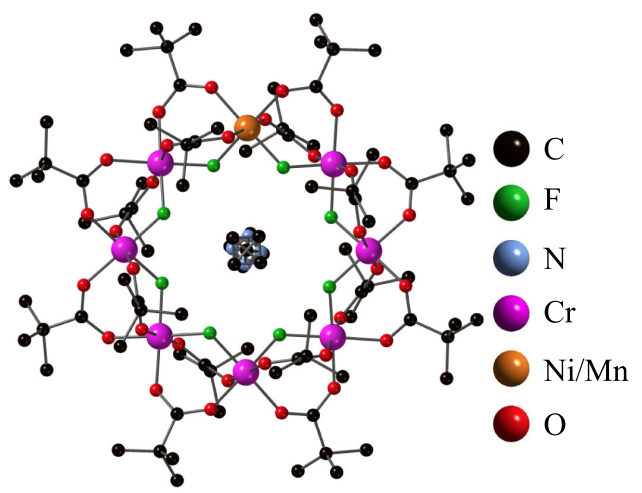

(b)

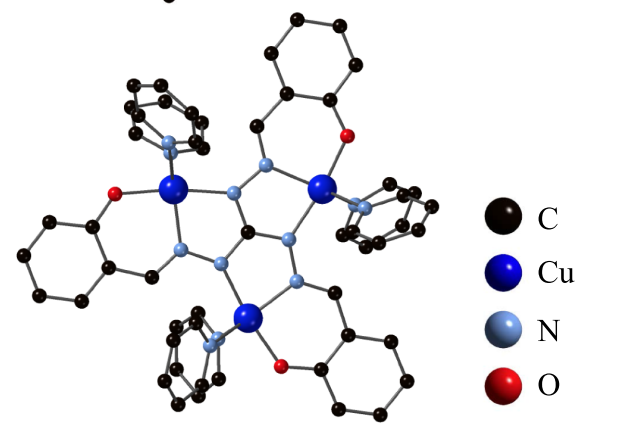

(c)

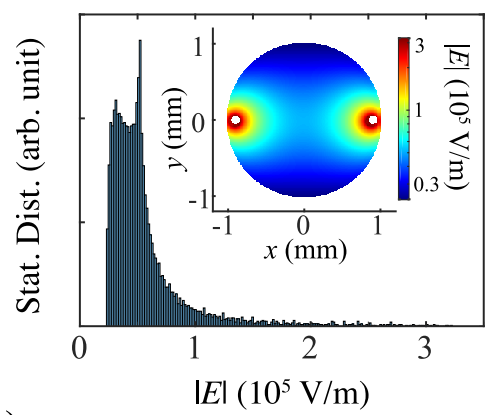

(e)

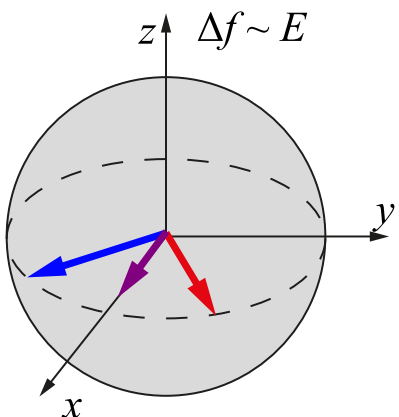

(d)

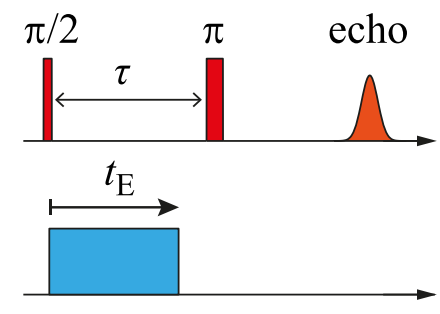

(f)

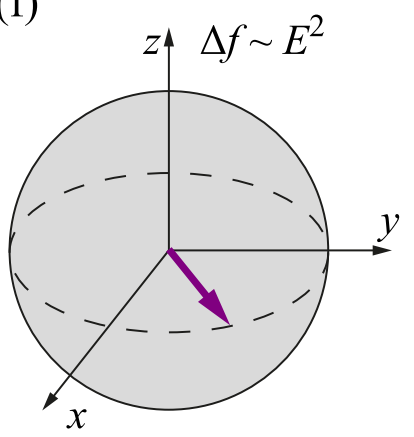

FIG. 1. (a) The molecular structure of the antiferromagnetic rings $\mathrm{Cr}_{7} \mathrm{Ni}$ and $\mathrm{Cr}_{7} \mathrm{Mn}$, which exhibit spin ground states of $S=1 / 2$ and $S=1$, respectively. (b) The molecular structure of the spin-frustrated $\mathrm{Cu}_{3}$, which exhibits a ground state with $S=1 / 2$. (c) Calculated distribution for the amplitude of the $E$ field $|E|$ in the ESR tube with $180 \mathrm{~V}$ applied across the electrodes. The color map illustrates the spatial distribution of $|E|$ in the cross section of the sample tube. (d) Schematic of the $E$-field experimental pulse sequence. A standard Hahn-echo sequence is employed to measure the spin echo signal. An $E$-field square pulse of duration $t_{E}$ is applied immediately after the $\pi / 2$ microwave pulse. The echo signal is recorded as a function of the $E$-field pulse duration to measure the spin-electric coupling effect. (e) and (f) illustrate the distinguishable effects of linear and quadratic coupling between the $E$ field and spins in an orientationally disordered molecular ensemble. The red and blue arrows in (e) represent the spins of molecules whose orientations are inverted with respect to each other, following a period of free precession under an $E$ field; a linear electric field effect gives rise to opposite phase shifts. The echo signal, which results from the sum of all spin packets, remains parallel to the $x$ axis in the rotating frame. For $\Delta f_{E} \propto E^{2}$, the phase shifts for molecules with inverted alignments are identical. Hence, the echo, indicated by the purple arrow in (f), shifts away from the $x$ axis in the rotating frame.

are dissolved in deuterated toluene, in order to extend the spin coherence at low temperatures. The $\mathrm{Cu}_{3}$ molecule [32], shown in Fig. 1(b), is similar to that reported in Ref. [33], with the bipyridine ligands replaced by pyridine. The molecules are dissolved in deuterated pyridine, which exchanges rapidly with the molecular pyridines, extending the spin coherence at low temperatures. The $\mathrm{Cu}_{3}$ core exhibits strong antiferromagnetic interactions between the $\mathrm{Cu}$ (II) ions, leading to a $S=1 / 2$ ground state at temperatures below $50 \mathrm{~K}$. The structures of all three molecules break inversion symmetry, so they can each, in principle, show a first order $E$-field effect [34].

We made ESR measurements using a commercial Bruker Elexsys $580 \mathrm{X}$-band pulsed ESR spectrometer, equipped with a ${ }^{4} \mathrm{He}$ flow cryostat for temperature control. The dissolved samples are contained in standard $3 \mathrm{~mm}$ diameter quartz ESR tubes equipped with a pair of electrode wires separated by about $1.8 \mathrm{~mm}$ and oriented parallel to the microwave magnetic field, in order to minimize the perturbation to the resonator. To aid impedance matching to the Avtech AVR-4-B voltage pulse generator, the electrodes are shorted above the microwave resonator by a $50 \Omega$ load, permitting square voltage pulses of up to $180 \mathrm{~V}$ with approximately $15 \mathrm{~ns}$ rise and fall times, durations up to $30 \mu \mathrm{s}$ in $200 \mathrm{~ns}$ steps, and a duty cycle of $0.5 \%$. This electrode geometry, immersed in the sample solution, generates an inhomogeneous $E$ field mostly perpendicular to the microwave magnetic field. The distribution of $E$-field strengths is shown in Fig. 1(c); when $180 \mathrm{~V}$ is applied to the electrodes, most of the sample experiences a field of between $2.5 \times 10^{4}$ and $6 \times 10^{4} \mathrm{~V} / \mathrm{m}$.

The pulse sequence design, shown in Fig. 1(d), is similar to that developed by Mims [34]. An E-field pulse is applied to the sample immediately after the $\pi / 2$ pulse in a standard Hahn-echo sequence. If a particular spin packet interacts with the $E$ field such that the ESR transition frequency is shifted by $\Delta f_{E}$, the spins accumulate an extra phase during free precession of $\Delta \varphi_{E}=2 \pi \Delta f_{E} t_{E}$. The inhomogeneity in the $E$ field leads to a distribution of phases accumulated across the sample. 

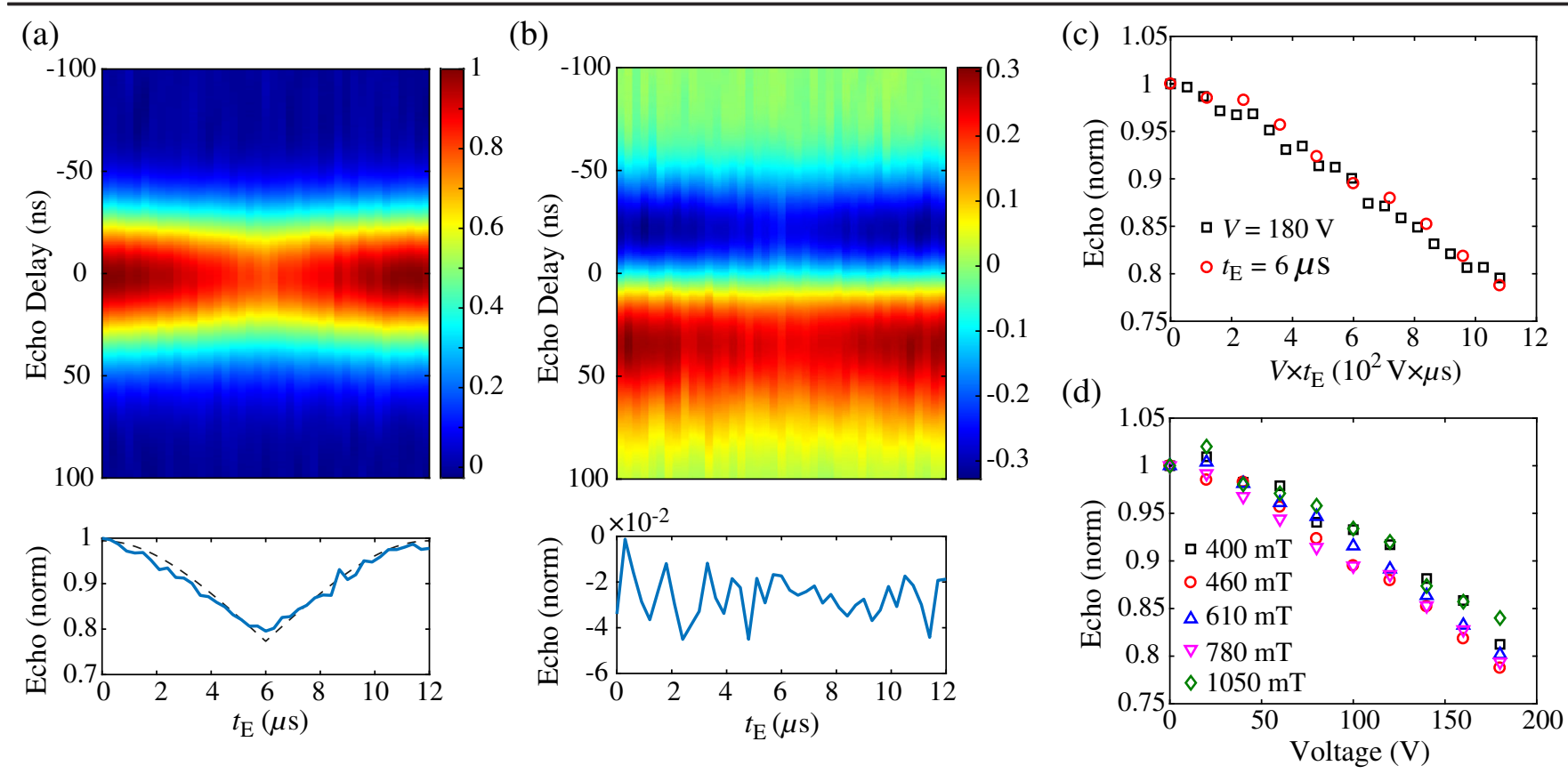

FIG. 2. $\quad E$-field effect on spin echoes for $\mathrm{Cr}_{7} \mathrm{Mn}$. (a) and (b), respectively, show the transient in-phase and out-of-phase echoes (color maps in upper panels) and the integrated echo intensities (lower panels) as a function of the duration of the $E$-field pulse (generated by applying a voltage of $180 \mathrm{~V}$ to electrodes immersed in the frozen solution). (c) Spin echo intensity as a function of the $E$-field pulse. The $E$-field pulse (abscissa) is defined as the applied voltage multiplied by its duration. The black squares correspond to measurements with fixed applied voltage $(180 \mathrm{~V})$ and varying $E$-field pulse duration $t_{E}$. The red circles are recorded for a fixed $t_{E}=6 \mu$ s while varying the $E$-field amplitude. (d) Dependence of the in-phase integrated echo on $E$ field measured at a range of magnetic field positions across the $\mathrm{Cr}_{7} \mathrm{Mn}$ ESR absorption spectrum. The temperature is $3 \mathrm{~K}$ throughout.

There is a further important contribution to the inhomogeneity of the response across the sample, arising from the fact that the molecules' orientations are distributed randomly in the frozen solution. In general, the sensitivity of the molecular spin Hamiltonian depends on the orientation of the molecule with respect to the $E$ field. The frequency shift induced on the spin of a molecule with orientation $\mathbf{n}$ is $\Delta f_{E}=2 \pi \mathbf{n} \cdot \overleftrightarrow{A} \cdot \mathbf{E}$, where $\overleftrightarrow{A}$ is a secondorder tensor describing the spin-electric coupling.

Inverting the direction of the $E$ field reverses the sign of the phase accumulated as a result of the $E$-field pulse. Thus for every spin packet gaining a phase $\Delta \varphi_{E}$ in response to the $E$-field pulse, there is another spin packet that is shifted by a phase $-\Delta \varphi_{E}$, as shown in Fig. 1(e).

Our experiment measures the ensemble response of the sample, integrated over all molecular orientations excited by the Hahn echo pulses and over the $E$-field distribution. The overall effect, for $t_{E}<\tau$, is to reduce the echo amplitude as a function of the $E$-field pulse duration and amplitude, without inducing an out-of-phase component to the echo [Fig. 1(e)]. The fact that we measure an averaged response means that we cannot extract from this experiment the detailed structure of $\overleftrightarrow{A}$. (This would require a sample with orientational order, i.e., a crystal; usually, though, dipolar couplings in crystals destroy the phase coherence required for the Hahn echo [1].) Instead, we characterize the response of each of the samples studied by an "average" isotropic response to the $E$ field, and this is the sensitivity figure that we quote.

A second-order coupling, i.e., $\Delta f_{E} \sim E^{2}$, leads to the same sign of $\Delta f_{E}$ across the ensemble [see Fig. 1(f)] and therefore a shift of the phase of the spin echo and an out-ofphase component to the echo. We can also check whether the coupling is linear or quadratic by studying the effects of $E$-field pulses of different amplitudes; if the coupling is linear, the response should depend only to the product $E t_{E}$.

Figure 2 shows typical data obtained from $\mathrm{Cr}_{7} \mathrm{Mn}$. Figures 2(a) and 2(b) show, respectively, the in-phase and out-of-phase components of the echoes as a function of the duration of the $E$-field pulse. The full transients are shown as color maps in the upper panels and the integrated components of the echoes are plotted in the lower panels. The echo signal is centered at zero delay time, with each vertical cut in the color maps representing an echo transient for $t_{E}$ given on the horizontal axis. There is a pronounced modulation of both echo components upon application of the $E$-field pulse visible in the transients. The integrated inphase echo shows a monotonic decrease as $t_{E}$, the duration of the $E$-field pulse increases from zero towards $\tau=6 \mu \mathrm{s}$, where the echo reaches $0.79 \pm 0.02$ of its $t_{E}=0$ value. The echo subsequently recovers as $t_{E}$ exceeds $\tau$ and approaches $2 \tau=12 \mu \mathrm{s}$, because the phase induced by the $E$ field during the first period of free evolution is progressively 
refocused following the Hahn echo $\pi$ pulse. This confirms that the effect of the $E$ field on the molecular spin is coherent. The integrated out-of-phase echo is insignificant throughout. These features are indicative of a linear coupling between the $\mathrm{Cr}_{7} \mathrm{Mn}$ spin and the applied $E$ field. Figure 2(c) shows that the echo intensity depends only on the product $V t_{E}$, confirming that the spin-electric coupling is linear.

The dashed line in the lower panel of Fig. 2(a) is a fit of the echo intensity given the $E$-field distribution shown in Fig. 1(c) assuming an isotropic $E$-field sensitivity $A$, yielding $A=1.9 \pm 0.1 \mathrm{rad} \mathrm{s}^{-1} / \mathrm{V} \mathrm{m}^{-1}$. This corresponds to an average phase shift across the ensemble of $\Delta \varphi=$ $\arccos (0.79) \approx 0.66 \mathrm{rad}$, implying an averaged $\Delta f=$ $\Delta \varphi /(2 \pi \tau)=17 \mathrm{kHz}$, or about $1.8 \times 10^{-6}$ of the ESR microwave frequency $(9.5 \mathrm{GHz})$.

The $S=1 \mathrm{Cr}_{7} \mathrm{Mn}$ spin exhibits a substantial anisotropy (with an axial term of about $20 \mathrm{GHz}$ and a rhombic term about an order of magnitude smaller [1]) such that at 9.5 GHz, the ESR spectrum of the disoriented ensemble extends from about $0.35 \mathrm{~T}$ to a little over $1 \mathrm{~T}$. Figure 2(d) shows the dependence of the integrated in-phase echo on voltage for a fixed $t_{E}$ measured at several points in the spectrum (corresponding to orientational subpopulations). When normalized to the amplitude of the echo in the absence of an $E$-field pulse, we find that there is only a weak dependence on magnetic field. In principle, this type of measurement allows us to separate the dependence of different spin-Hamiltonian terms on the $E$ field. However, the field dependence that we measure in our disoriented ensemble is not sufficiently distinctive to do this reliably; measurements on oriented ensembles would provide stronger data.

Experiments on $\mathrm{Cu}_{3}$ revealed a comparable dependence on the $E$ field [see Fig. 3(a)], with the normalized echo decreasing to $0.85 \pm 0.03$ of its $t_{E}=0$ value for an $E$-field pulse of $t_{E}=5 \mu \mathrm{s}$. Fitting the echo amplitude [as above, the dashed line in the lower panel of Fig. 3(a)] yields a spinelectric coupling of $A=1.9 \pm 0.2 \mathrm{rad} \mathrm{s}^{-1} / \mathrm{V} \mathrm{m}^{-1}$.

The data from $\mathrm{Cr}_{7} \mathrm{Ni}$ [Fig. 3(b)] show no evidence of spin-electric coupling. The lack of an effect in $\mathrm{Cr}_{7} \mathrm{Ni}$, which shares its broken-inversion-symmetry structure with $\mathrm{Cr}_{7} \mathrm{Mn}$, is interesting. The key difference between $\mathrm{Cr}_{7} \mathrm{Ni}$ and $\mathrm{Cr}_{7} \mathrm{Mn}$ is the total spin in the ground state $(S=1 / 2$ and $S=1$, respectively). This suggests that the axial and rhombic zero field splitting anisotropy terms, relevant for $\mathrm{Cr}_{7} \mathrm{Mn}$ but not for $\mathrm{Cr}_{7} \mathrm{Ni}$, may be important in offering a sensitivity to the $E$ field, as was found, e.g., in $\mathrm{Mn}$ defects in $\mathrm{ZnO}$ [25]. (We note here that the other contribution to the spin anisotropy, through the $g$ factor, is rather weak in both $\mathrm{Cr}_{7} \mathrm{Ni}$ and $\mathrm{Cr}_{7} \mathrm{Mn}$.) In this picture, the $E$-field sensitivity exhibited by $\mathrm{Cu}_{3}$ might be associated with the magnetic frustration inherent in its structure, as proposed by Trif et al. $[18,19]$. On the other hand, we note the strength of the $E$-field effects in $\mathrm{Cr}_{7} \mathrm{Mn}$ and $\mathrm{Cu}_{3}$ are (a)

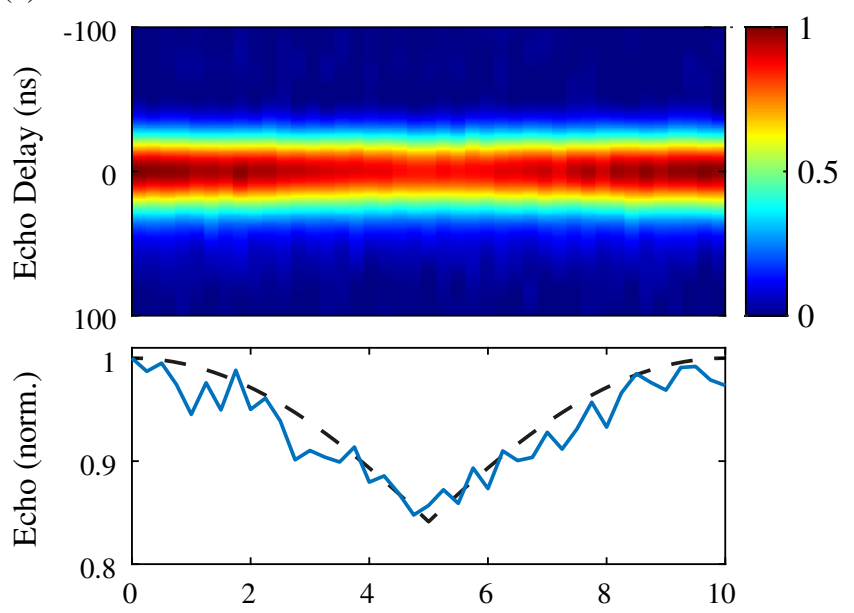

(b)
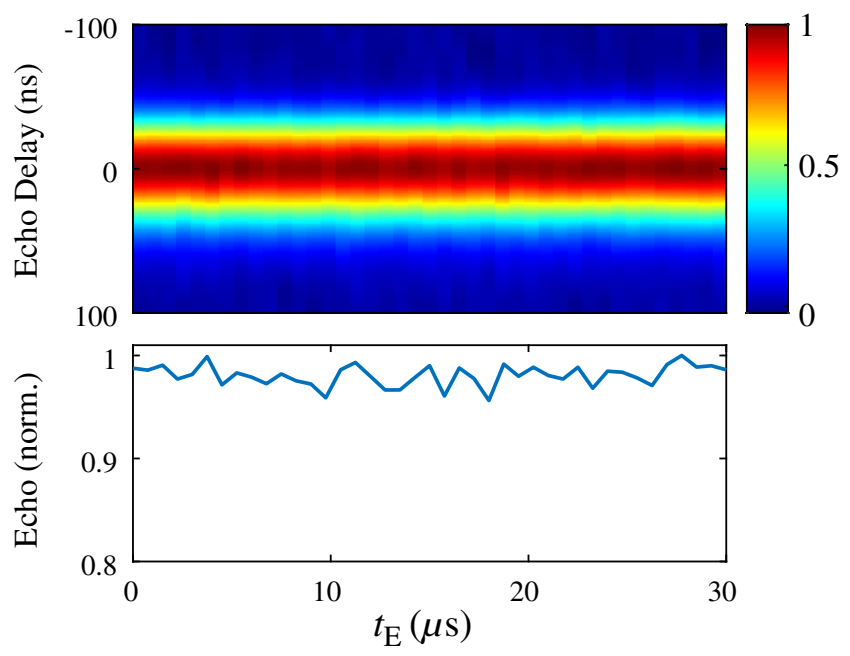

FIG. 3. The in-phase transient (upper) and integrated (lower) echoes for (a) $\mathrm{Cu}_{3}$ and (b) $\mathrm{Cr}_{7} \mathrm{Ni}$ as a function of the duration of the $E$ field. The amplitude of the $E$-field pulses is fixed at $180 \mathrm{~V}$ in both cases. $\tau=5$ and $15 \mu$ s in the microwave Hahn-echo sequences for $\mathrm{Cu}_{3}$ and $\mathrm{Cr}_{7} \mathrm{Ni}$, respectively. The temperature is $3 \mathrm{~K}$.

comparable to the Stark effect in P donors in Si [26], where a quadratic $E$-field sensitivity enters through the hyperfine interaction. In principle, $a b$ initio methods can help to explain and predict the magnitude of the $E$-field sensitivity [19] as shown in Ref. [20] for a different $\mathrm{Cu}_{3}$ molecular cluster [35].

The magnitudes of the spin-electric couplings that we observe in $\mathrm{Cr}_{7} \mathrm{Mn}$ and $\mathrm{Cu}_{3}$ are such that it would be challenging to use them for direct manipulation of the spin via microwave modulation of the $E$ field [25,28], but they could be adequate for tuning the ESR transition frequency on short timescales (cf. the "A gate" proposed by Kane for $\mathrm{P}$ donors in $\mathrm{Si}[17,36])$. For example, with sufficiently localized electrodes, the resonance frequency of an individual $\mathrm{Cr}_{7} \mathrm{Mn}$ molecule can be shifted by $\sim 32 \mathrm{MHz}$ with an $E$ field of $10^{8} \mathrm{~V} / \mathrm{m}$, an $E$ field routinely accessible in 
reported molecular break-junction devices [8,9]. Such control of the resonance frequency is sufficient to shift the molecule in or out of resonance with a globally applied $30 \mathrm{~ns}$ microwave pulse, 100 times shorter than the coherence time of $\mathrm{Cr}_{7} \mathrm{Mn}$ molecules. This would fulfil the requirement of selectively controlling individual molecules while achieving rapid spin manipulation using globally applied microwave pulses.

The spin-electric coupling in molecular nanomagnets might be enhanced through prudent choice of molecular spin centers. For example, rare earth ions with large spinorbit or hyperfine interactions exhibit sufficient couplings to achieve coherent $E$-field-driven manipulations [9,22]. Alternatively, designing exchange-coupled metal clusters with significant electric dipole moments might offer a route to $E$-field tuning of the intramolecular exchange interactions, and therefore $E$-field control over ground-state spin properties. Ab initio methods can help to guide such rational design [20], e.g., by identifying intramolecular exchange interactions that are particularly sensitive to externally applied $E$ fields. The experimental method reported here will be important in offering rapid screening of $E$-field sensitivities.

We note that a study of the $E$-field effect on the continuous-wave ESR spectrum of a crystal of a polynuclear molecular nanomagnet was reported after the submission of this Letter [37].

We thank EPSRC (Grants No. EP/L011972/1, No. EP/ P000479/1, No. EP/R043701/1), the European Project SUMO (QuantERA call 2017), and European COST Action CA15128 MOLSPIN for support. J. M. is supported by Magdalen College, Oxford.

*junjie.liu@physics.ox.ac.uk †arzhang.ardavan@physics.ox.ac.uk

[1] A. Ardavan, O. Rival, J. J. L. Morton, S. J. Blundell, A. M. Tyryshkin, G. A. Timco, and R. E. P. Winpenny, Phys. Rev. Lett. 98, 057201 (2007).

[2] C. J. Wedge, G. A. Timco, E. T. Spielberg, R. E. George, F. Tuna, S. Rigby, E. J. L. McInnes, R. E. P. Winpenny, S. J. Blundell, and A. Ardavan, Phys. Rev. Lett. 108, 107204 (2012).

[3] J. M. Zadrozny, J. Niklas, O. G. Poluektov, and D. E. Freedman, ACS Cent. Sci. 1, 488 (2015).

[4] M. Atzori, E. Morra, L. Tesi, A. Albino, M. Chiesa, L. Sorace, and R. Sessoli, J. Am. Chem. Soc. 138, 11234 (2016).

[5] M. Shiddiq, D. Komijani, Y. Duan, A. Gaita-Ariño, E. Coronado, and S. Hill, Nature (London) 531, 348 (2016).

[6] A. Fernandez, J. Ferrando-Soria, E. M. Pineda, F. Tuna, I. J. Vitorica-Yrezabal, C. Knappke, J. Ujma, C. A. Muryn, G. A. Timco, P. E. Barran, A. Ardavan, and R. E. Winpenny, Nat. Commun. 7, 10240 (2016).

[7] A. Ardavan, A. M. Bowen, A. Fernandez, A. J. Fielding, D. Kaminski, F. Moro, C. A. Muryn, M. D. Wise, A. Ruggi,
E. J. L. McInnes, K. Severin, G. A. Timco, C. R. Timmel, F. Tuna, G. F. S. Whitehead, and R. E. P. Winpenny, npj Quantum Inf. 1, 15012 (2015).

[8] E. Burzurí, A. S. Zyazin, A. Cornia, and H. S. J. van der Zant, Phys. Rev. Lett. 109, 147203 (2012).

[9] S. Thiele, F. Balestro, R. Ballou, S. Klyatskaya, M. Ruben, and W. Wernsdorfer, Science 344, 1135 (2014).

[10] M. Misiorny, E. Burzurí, R. Gaudenzi, K. Park, M. Leijnse, M. R. Wegewijs, J. Paaske, A. Cornia, and H. S. J. van der Zant, Phys. Rev. B 91, 035442 (2015).

[11] E. Moreno-Pineda, C. Godfrin, F. Balestro, W. Wernsdorfer, and M. Ruben, Chem. Soc. Rev. 47, 501 (2018).

[12] M. N. Leuenberger and D. Loss, Nature (London) 410, 789 (2001).

[13] F. Troiani, M. Affronte, S. Carretta, P. Santini, and G. Amoretti, Phys. Rev. Lett. 94, 190501 (2005).

[14] J. Lehmann, A. Gaita-Ariño, E. Coronado, and D. Loss, Nat. Nanotechnol. 2, 312 (2007).

[15] L. Bogani and W. Wernsdorfer, Nat. Mater. 7, 179 (2008).

[16] A. Schweiger and G. Jeschke, Principles of Pulse Electron Paramagnetic Resonance (Oxford University Press, New York, 2001).

[17] B. E. Kane, Nature (London) 393, 133 (1998).

[18] M. Trif, F. Troiani, D. Stepanenko, and D. Loss, Phys. Rev. Lett. 101, 217201 (2008).

[19] M. Trif, F. Troiani, D. Stepanenko, and D. Loss, Phys. Rev. B 82, 045429 (2010).

[20] M. F. Islam, J. F. Nossa, C. M. Canali, and M. Pederson, Phys. Rev. B 82, 155446 (2010).

[21] J. J. Pla, K. Y. Tan, J. P. Dehollain, W. H. Lim, J. J. L. Morton, D. N. Jamieson, A. S. Dzurak, and A. Morello, Nature (London) 489, 541 (2012).

[22] C. Godfrin, A. Ferhat, R. Ballou, S. Klyatskaya, M. Ruben, W. Wernsdorfer, and F. Balestro, Phys. Rev. Lett. 119, 187702 (2017).

[23] V. N. Golovach, M. Borhani, and D. Loss, Phys. Rev. B 74, 165319 (2006).

[24] W. Mims, The Linear Electric Field Effect in Paramagnetic Resonance (Oxford University Press, Oxford, 1976).

[25] R. E. George, J. P. Edwards, and A. Ardavan, Phys. Rev. Lett. 110, 027601 (2013).

[26] F. R. Bradbury, A. M. Tyryshkin, G. Sabouret, J. Bokor, T. Schenkel, and S. A. Lyon, Phys. Rev. Lett. 97, 176404 (2006)

[27] A. J. Sigillito, A. M. Tyryshkin, and S. A. Lyon, Phys. Rev. Lett. 114, 217601 (2015).

[28] S. Baumann, W. Paul, T. Choi, C. P. Lutz, A. Ardavan, and A. J. Heinrich, Science 350, 417 (2015).

[29] M. Affronte, S. Carretta, G. A. Timco, and R. E. P. Winpenny, Chem. Commun., 1789 (2007).

[30] G. A. Timco, E. J. L. McInnes, and R. E. P. Winpenny, Chem. Soc. Rev. 42, 1796 (2013).

[31] F. K. Larsen, E. J. L. McInnes, H. E. Mkami, J. Overgaard, S. Piligkos, G. Rajaraman, E. Rentschler, A. A. Smith, G. M. Smith, V. Boote, M. Jennings, G. A. Timco, and R. E. P. Winpenny, Angew. Chem. 42, 101 (2003).

[32] B. Kintzel, M. Böhme, J. Liu, A. Burkhardt, J. Mrozek, A. Buchholz, A. Ardavan, and W. Plass, Chem. Commun. 54, 12934 (2018). 
[33] E. T. Spielberg, A. Gilb, D. Plaul, D. Geibig, D. Hornig, D. Schuch, A. Buchholz, A. Ardavan, and W. Plass, Inorg. Chem. 54, 3432 (2015).

[34] W. B. Mims, Rev. Sci. Instrum. 45, 1583 (1974).

[35] Note that the results of Ref. [20] are not directly applicable here because the $E$-field effect depends sensitively on the details of the molecular structures, which differ substantially.
[36] A. Laucht, J. T. Muhonen, F. A. Mohiyaddin, R. Kalra, J. P. Dehollain, S. Freer, F. E. Hudson, M. Veldhorst, R. Rahman, G. Klimeck, K. M. Itoh, D. N. Jamieson, J. C. McCallum, A. S. Dzurak, and A. Morello, Sci. Adv. 1, e1500022 (2015).

[37] A. K. Boudalis, J. Robert, and P. Turek, Chem. Eur. J. 24, 14896 (2018). 